\title{
Phase-Matching for Generation of Isolated Attosecond XUV and Soft-X-Ray Pulses with Few-Cycle Drivers
}

\author{
J. Schötz, ${ }^{1,2, *}$ B. Förg, ${ }^{1,2}$ W. Schweinberger, ${ }^{1,3}$ I. Liontos, ${ }^{3}$ H. A. Masood $\odot,{ }^{3}$ A. M. Kamal, ${ }^{3}$ C. Jakubeit, ${ }^{2}$ N. G. Kling $\odot,{ }^{1}$ \\ T. Paasch-Colberg, ${ }^{1,3}$ S. Biswas $\odot,{ }^{1,2}$ M. Högner ${ }^{1,2}$ I. Pupeza, ${ }^{1,2}$ M. Alharbi, ${ }^{3}$ A. M. Azzeer $\odot,{ }^{3, \dagger}$ and M. F. Kling $\oplus^{1,2, ;}$ \\ ${ }^{1}$ Physics Department, Ludwig-Maximilians-Universität Munich, D-85748 Garching, Germany \\ ${ }^{2}$ Max Planck Institute of Quantum Optics, D-85748 Garching, Germany \\ ${ }^{3}$ Attosecond Science Laboratory, Physics and Astronomy Department, \\ King Saud University, Riyadh, Saudi Arabia
}

(Received 18 December 2019; revised 2 July 2020; accepted 19 August 2020; published 15 October 2020)

\begin{abstract}
Isolated attosecond pulses (IAPs) produced through laser-driven high-harmonic generation (HHG) hold promise for unprecedented insight into physical, chemical, and biological processes via attosecond $\mathrm{x}$-ray diffraction and spectroscopy with tabletop sources. Efficient scaling of HHG towards X-ray energies, however, has been hampered by ionization-induced plasma generation impeding the coherent buildup of high-harmonic radiation. Recently, it has been shown that these limitations can be overcome in the socalled "overdriven regime" where ionization loss and plasma dispersion strongly modify the driving laser pulse over small distances, albeit without demonstrating IAPs. Here, we report on experiments contrasting the generation of IAPs at $80 \mathrm{eV}$ in argon with neon via attosecond streaking measurements. Comparing our experimental results to numerical simulations, we conclude that IAPs in argon are generated in the overdriven regime. We introduce a simple expression that fully describes the HHG dipole phase-mismatch contribution, specifically the effect of the blueshift of the driving laser. Furthermore, we present a method to numerically calculate the transient HHG phase mismatch, which allows us to demonstrate the accuracy of the introduced phase-mismatch expression. Finally, we perform simulations for different gases and wavelengths and show that including the full HHG dipole phase-mismatch contribution is important for understanding HHG with long-wavelength, few-cycle laser pulses in high-pressure gas targets, which are currently being employed for scaling isolated attosecond pulse generation beyond extreme ultraviolet (XUV) towards soft-X-ray photon energies.
\end{abstract}

DOI: 10.1103/PhysRevX.10.041011

\section{INTRODUCTION}

High-harmonic generation (HHG) in atoms or molecules [1-4] constitutes the building block for the foundation of attosecond science [5-10]. It arises when an intense laser field interacts with an atom or molecule, and it can be understood by an intuitive semiclassical model [11] describing the process in three steps: An electron tunnels out of the atom through the potential barrier; it subsequently propagates under the influence of the electric field; and finally, it recollides and recombines with the

\footnotetext{
*johannes.schoetz@mpq.mpg.de

azzeer@ksu.edu.sa

*matthias.kling@lmu.de
}

Published by the American Physical Society under the terms of the Creative Commons Attribution 4.0 International license. Further distribution of this work must maintain attribution to the author(s) and the published article's title, journal citation, and DOI. Open access publication funded by the Max Planck Society.
Subject Areas: Atomic and Molecular Physics,

Photonics atom, generating a photon whose energy typically lies in the extreme ultraviolet (XUV, $10 \mathrm{eV}-100 \mathrm{eV}$ ) or soft-x-ray region $(100 \mathrm{eV}-1 \mathrm{keV})$. Further advances in the area of subcycle waveform control of laser pulses [12,13], in combination with sophisticated techniques developed to exploit the intrinsic synchronization between the HHG radiation $[7,11]$ and the driving laser field, have led to the production of isolated attosecond pulses (IAPs) [6]. Generation of IAPs extended the powerful techniques of pump-probe spectroscopy to the electronic time scale and paved the way to a plethora of applications.

In order to extract single IAPs from HHG radiation, several gating schemes have been proposed and demonstrated experimentally [9]. Apart from amplitude gating [6], polarization gating [14], double optical gating [15], or interferometric polarization gating [16] achieved by shaping the waveform of the laser pulses, controlling the ionization of the HHG target gaseous medium provides two more ways to generate IAPs. First, by using a strong enough field, the ground state of the atom can be depleted 
by a single half-cycle in the leading edge of a laser field, inhibiting XUV emission during later half-cycles $[17,18]$. Second, the ionization of atoms leads to an additional timedependent dispersive plasma term for the driving laser pulse, such that phase-matching between the driving pulse and generated $\mathrm{HHG}$ can be confined to a single half-cycle [19-24], which is termed transient phase-matching. This method has recently sparked a lot of interest in HHG and especially single IAP generation with long-wavelength driving laser pulses that can reach up to $1-\mathrm{keV}$ photon energy spanning the water window $[25,26]$.

In HHG, the phase mismatch not only consists of the wave-vector mismatch of the generated harmonic radiation and the driving laser, but it also contains a contribution due to the phase of the HHG dipole, which depends on the intensity and wavelength of the driving laser [27]. If the driving laser changes only slowly within the HHG target, the dipole contribution can be ignored. Perfect phasematching is reached at a critical ionization fraction $\eta_{\mathrm{cr}}$ [28] of the HHG medium, when the plasma-induced dispersion and the atomic dispersion balance. For a given laser-pulse shape, $\eta_{\mathrm{cr}}$ determines the maximum intensity for which phase-matching at the peak of the pulse is possible, thereby limiting the maximum XUV energy that can be phase-matched. This maximum photon energy has been termed the phase-matching cutoff [28], and this picture is commonly used in the discussion of waveguide-based HHG sources [24,25,28-30].

With the use of longer-wavelength driver lasers to achieve a higher cutoff in the water window and beyond, higher gas pressure is required in order to compensate for the lower single-atom HHG conversion efficiency [26,29,31]. However, this higher gas pressure leads to a higher plasma density and, consequently, a reshaping of the driving laser pulse, which in gas targets occurs mostly through plasma defocusing, resulting in an intensity reduction and blueshift. The latter is most pronounced for few-cycle pulses. This pulse reshaping affects HHG phase-matching, and the dipole contribution has to be taken into account, which has been the subject of a number of theoretical [31-34] and experimental [35-38] studies.

Several important aspects about HHG in this regime have been revealed theoretically. Generally, since the phase mismatch is proportional to the harmonic order, for high photon energies and a driving pulse undergoing reshaping, perfect phase-matching can only be achieved locally and transiently. Furthermore, the HHG dipole phase can counteract the plasma-induced phase increase of the driving laser, either through a blueshift [32] or an intensity decrease [33], leading to perfect phase-matching and XUV buildup over propagation lengths of $10-100 \mu \mathrm{m}$. While the former has been used to explain experiments with an XUV cutoff significantly above the phase-matching cutoff up to the $\mathrm{keV}$ energy range [39-42], due to the low photon flux, it is not clear whether perfect phase-matching has been achieved. Finally, simulations of HHG with long-wavelength drivers and high-pressure gas targets with realistic pressure distributions have shown a pronounced intensity clamping of the driving laser [31].

On the experimental front, there have been several studies involving long-wavelength drivers or tight-focusing conditions or both $[35,36,38]$, including one with an additional plasma density measurement [37], indicating the importance of plasma defocusing. In the latter [37], the simulations showed XUV buildup over a few $100 \mu \mathrm{m}$, an intensity clamping, and plasma-induced pulse reshaping of the driving laser. This regime was termed the "overdriven regime."

However, while the intensity decay is usually included in the analysis, none of these studies has presented an expression for the phase mismatch that explicitly contains the blueshift contribution to the dipole phase. Indeed, except for the 1D simulations in Refs. [32,43], the latter is usually ignored. Moreover, the experiments summarized above only measured XUV spectra and did not directly temporally characterize the IAPs and driving laser pulses.

Here, we study the generation of IAPs in neon and argon, at $80-\mathrm{eV}$ photon energy driven by few-cycle laser pulses at a 750-nm central wavelength and with intensity around $5 \times 10^{14} \mathrm{~W} / \mathrm{cm}^{2}$, both experimentally and numerically. The complete temporal characterization of the IAPs and the laser pulses with attosecond streaking [44-47] suggests that while the results in neon are in agreement with the amplitude gating scheme, the IAPs from argon cannot be understood by employing the same scheme within the single-atom picture. This discrepancy is caused by phase-matching effects. Here, the simulations and their excellent agreement with the experimental findings show that IAPs are generated above the classical phase-matching cutoff in the overdriven regime, where the driving laser pulses are strongly modified and the plasma-induced transient phase-matching is responsible for the gating of the IAPs. To be able to describe this case, we introduce an extended analytic phase-matching expression that fully includes the effect of the strong plasma-induced driving pulse reshaping on the HHG dipole phase, especially the blueshift. Moreover, we present a simple method to numerically calculate the transient phase mismatch, which helps us to show the accuracy of our phase-matching expression. Through simulations, our findings are verified for different wavelengths and gas types under strong focusing conditions. We show that at the conditions of maximized XUV photon flux, the contribution of the blueshift to the dipole phase can be the dominant phasemismatch term. Our results offer a significantly improved understanding of HHG phase-matching with plasmareshaped few-cycle driving pulses, and they are of direct 
relevance to the current efforts to push HHG into the water window and beyond.

\section{RESULTS AND DISCUSSION}

\section{A. Experimental approach and theoretical simulations}

The experimental approach, which employs attosecond streaking spectroscopy, is illustrated schematically in Fig. 1(a) and described in detail in the Supplemental Material (SM) [48]. In short, few-cycle laser pulses covering the infrared (IR) wavelength range from $450 \mathrm{~nm}$ to
$1000 \mathrm{~nm}$ and centered around $750 \mathrm{~nm}$ are focused through a static gas target (neon or argon) for HHG. The spatially separated but concentric XUV and IR beams are focused again with a two-component focusing mirror on a neon gas jet for streaking, where a time-of-flight (TOF) spectrometer is used for kinetic energy analysis of the photoelectrons. The inner part of the double mirror, which is movable with respect to the outer one for variable pump-probe time delay, reflects the XUV centered around $80 \mathrm{eV}$ with a bandwidth of $6.4 \mathrm{eV}$ and a flat spectral phase. We use variable HHG target gas pressure, the HHG focusing mirror position, and
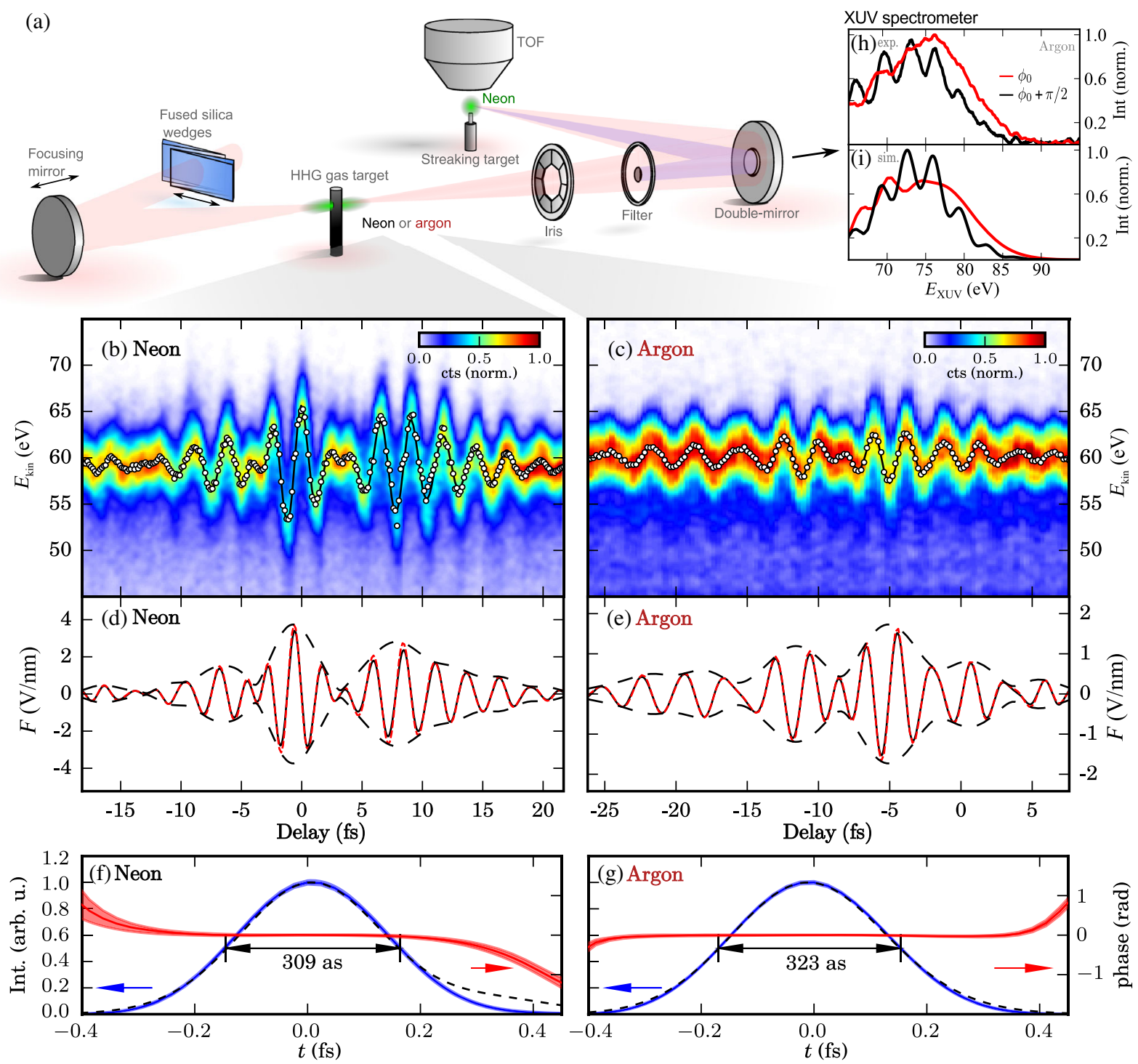

FIG. 1. HHG generation at $80 \mathrm{eV}$ with sub-two-cycle pulses at $750 \mathrm{~nm}$ in neon and argon. (a) Scheme of the experimental setup. (b, c) Experimental attosecond streaking traces for isolated attosecond pulse generation through HHG in neon and in argon, respectively. The retrieved raw (dots) and filtered (solid line) streaking curves from the Gaussian fits are shown. (d,e) Electric field reconstructed from the streaking curves from the Gaussian fit (red dashed line) and the ptychographic reconstruction algorithm (black solid line). (f, g) Reconstructed temporal profiles of XUV intensity (blue) and phase (red), for neon and argon, respectively. The black dashed line shows the pulses from the simulation. The shaded areas show the standard deviation (see text for details). (h,i) Experimental and simulated XUV spectra in argon when the double mirror is replaced with a grating spectrometer for two different values of the CEP (see Sec. IV for details). 
dispersion control by fused silica wedge insertion to optimize IAP generation (see Sec. IV for details on the optimization procedure). For example, when switching from neon to argon, additional dispersion and a shift of the focus position with respect to the target are needed. The HHG spectrum can also be recorded with an XUV spectrometer by removing the double mirror.

The attosecond streaking spectrograms are shown in Fig. 1 for XUV generated in neon (b) and argon (c), from which precise information including the electric field of the IR pulses, as shown in Figs. 1(d) and 1(e), at the streaking focus can be obtained $[44,49]$. Here, both spectrograms are normalized. Note, however, that the XUV photon flux for argon is roughly a factor of 3 lower than for neon. For a coarse reconstruction, a streaking curve is extracted from the central energy of the fit of a Gaussian function to the photoelectron spectrum for each delay step (white dots). This curve is subsequently smoothed by filtering out frequency components beyond the laser bandwidth.

In order to reconstruct the attosecond XUV pulses, we employ the ptychographic reconstruction algorithm described in Ref. [50]. As can be seen in Figs. 1(f) and $1(\mathrm{~g})$, the reconstruction yields XUV temporal profiles (blue lines) for argon and neon HHG with $309 \pm 9$ as and $323 \pm 4$ as full-width-at-half-maximum (FWHM) duration, respectively, with almost negligible chirp and almost no satellite pulses (contrast better than 50:1) as is also evident from the shape of the spectrograms. The algorithm furthermore returns the streaking laser pulse as shown in Figs. 1(d) and 1(e) in black. Both streaking pulse shapes agree very well with the ones obtained through the Gaussian fits.

In order to gain insight into the pulse propagation dynamics in the HHG target, we perform numerical simulations with a model described in detail in Ref. [51]. In short, for both the driving field and the XUV field, linear refraction and absorption are considered in paraxial approximation with cylindrical symmetry. Moreover, for the driving laser field, the Kerr effect and the plasma generation-leading to ionization loss, plasma defocusing, and blueshift-are taken into account. For the XUV emission, the dipole response of individual atoms is modeled using the strong-field approximation (SFA) with hydrogenlike dipole moments, including ground-state depletion by a static ionization rate [52]. Even though the use of hydrogenlike dipole moments means that we cannot expect absolute quantitative agreement in terms of photon flux, phase-matching effects are adequately described within SFA. The input pulse for the simulations is obtained from the measured streaking trace in neon through a detailed modeling of the propagation through the HHG target and the beamline (see SM [48] for details).

The experimentally measured XUV spectrum in argon is shown in Fig. 1(h) and compared to simulations in Fig. 1(i). Good agreement between the experiment and simulation is observed, including details like the steplike increase of the spectrum for CEP $=\phi_{0}$ (red curves) at lower energies. With this agreement, the temporal behavior of the IAPs, as shown in Figs. 1(f) and 1(g), is very well reproduced, especially for argon. There is a slight overestimation of the trailing edge in the neon pulse shape, which might be due to an overestimated weight of long trajectories in SFA compared to full quantum mechanical simulations [27].

Two aspects of our experimental results are remarkable. First of all, the phase-matching cutoff for our pulses in argon should be around $70 \mathrm{eV}$. However, we observe IAPs in argon at $80 \mathrm{eV}$. Second, we observe a distinct difference in the measured streaking electric fields in Figs. 1(d) and 1(e). While the change in laser-pulse shapes in the two cases is mostly due to the spectral phase change caused by the additional fused silica wedge insertion for argon obtained from the experimental optimization, the carrierenvelope phase (CEP) of the measured waveforms differs by roughly $\pi / 2$. Even though the CEP $=0$ for neon might be a coincidence, the relative CEP shift of the argon streaking trace cannot be easily explained without assuming a considerably different CEP in the HHG target. We interpret both observations as a hint that the HHG dynamics in argon is different from neon, especially with regard to phase-matching.

\section{B. XUV generation in the overdriven regime}

Since transient phase-matching will affect how XUV radiation builds up from the XUV polarization, we start by comparing the XUV temporal profile and the corresponding source term. Figure 2(a) shows the spatiotemporally resolved XUV electric-field amplitude at the end of the argon HHG target within the XUV multilayer mirror bandwidth. The resulting pulse shows a strong peak at around $0.1 \mathrm{fs}$ and some very small, almost indiscernible, satellite pulses at around $-1.1 \mathrm{fs}$ and $1.3 \mathrm{fs}$ [better visualized in panel (c)]. The smooth radial profile seems to indicate that off-axis phase-matching does not play a major role here, as opposed to tighter focusing conditions [36].

To see whether and how the XUV buildup is influenced by any transient phase-matching, we compare the XUV field amplitude to the time-resolved HHG source term, shown in Fig. 2(b), in the same energy window. We calculate the latter for each spatial coordinate from the nonlinear polarization in the HHG target. From this calculation, the source signal, which would be generated from perfect, time-independent phase-matching, is determined by integrating the absolute value of the HHG source amplitude along the propagation axis. In comparison to the XUV temporal profile, the HHG source term exhibits, besides the main pulse at around $0.1 \mathrm{fs}$, another significant contribution about one half-cycle later, at around $1.3 \mathrm{fs}$, which is strongly suppressed in the XUV spatiotemporal structure. The suppression of the second pulse can only be 

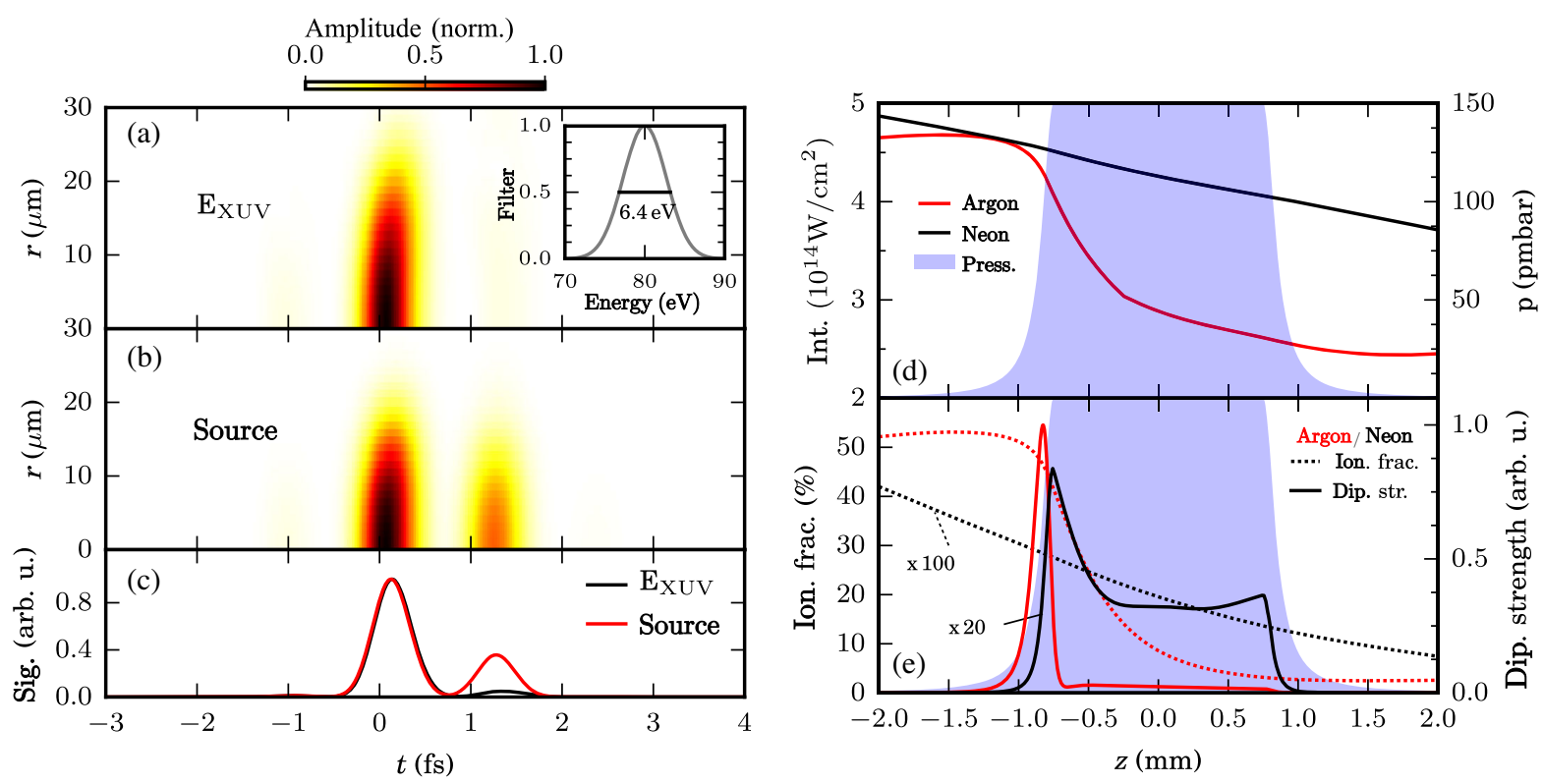

FIG. 2. Simulation of XUV generation in argon and evolution of the driving laser. (a) Spatiotemporal structure of XUV pulse at the end of the target calculated within the energy window illustrated by the inset, which shows an isolated attosecond pulse. (b) Spatiotemporal structure of the XUV source term within the above energy window integrated along the propagation axis assuming perfect phase-matching. (c) Comparison of the far-field XUV temporal profile at the position of the double mirror (solid black line) with the radially integrated source term from panel (b) (red). The absence of the satellite pulse at around 1.3 fs compared to the source term indicates that a transient phase-matching mechanism is present. (d) Evolution of the peak intensity of the driving laser pulse on the propagation axis within the gas target for neon (black line) and argon (red line). The blue shaded areas here and in panel (e) show the gas pressure distribution for both argon and neon. The propagation in argon is strongly influenced by the interaction with the gas. (e) XUV source term (solid line) within the energy region specified in the inset of panel (a) and the ionization fraction at the end of the pulse (dashed lines) for neon (black) and argon (red). The source term for argon is restricted to the entrance of the gas target. Note the different scaling for argon and neon.

explained by a time-dependent macroscopic phase mismatch. As a side remark, the HHG source term is more centered around the propagation axis compared to the XUV field, which is due to the neglect of diffraction. The suppression of the second pulse is further illustrated in Fig. 2(c), which shows the XUV electric field (black solid line) and HHG source (red solid line) integrated along the radial axis.

\section{Limitations of phase-matching descriptions without a dipole term in the overdriven regime}

In order to understand how different aspects of the pulse propagation affect the XUV generation and buildup, we need to briefly revise the general expression for phasematching. In the most general form, the phase mismatch $\Delta k\left(\omega_{q}\right)$ of the $q$ th harmonic at frequency $\omega_{q}$ is given by the difference between the wave vector $k$ and the phase gradient of the source term [27]:

$\Delta k\left(\omega_{q}\right)=k_{\omega_{q}}-\nabla \phi_{\text {source }}=k_{\omega_{q}}-q \cdot \nabla \phi_{\mathrm{IR}}-\nabla \phi_{\text {dipole }}$.

As shown above, the source term can be further decomposed into a contribution from the driving laser field $\phi_{\mathrm{IR}}$ and the intrinsic HHG dipole phase $\phi_{\text {dipole }}$. The former contains the Gouy phase as well as the wave vector of the driving laser field, which depends on the gas pressure and plasma density. The latter term, which is absent in perturbative harmonic generation, depends both on the driving laser intensity $I$ and frequency $\omega_{0}$ and can be approximated by $\nabla \phi_{\text {dipole }}=-\alpha_{j} \nabla\left(U_{\mathrm{p}} / \hbar \omega_{0}\right)$ [27], where $\alpha_{j}$ is an energy-dependent proportionality constant that differs for long and short trajectories and $U_{\mathrm{p}}$ is the ponderomotive potential. Since the driving laser can lead to a significant increase of plasma density between different half-cycles, which in turn influences the pulse itself, specifically its wave vector, the phase mismatch generally depends on time $t$.

As we are interested in the XUV far field close to the optical axis, and because there is, additionally, no indication of a particular off-axis contribution [see Fig. 2(a)], we restrict our analysis of the phase mismatch in the gas target to the propagation axis $(r=0)$ in the longitudinal direction $\left(\vec{k}_{\omega_{q}} \mid \vec{e}_{z}\right)$ and to the energy window around $80 \mathrm{eV}$, unless stated otherwise.

For a driving laser pulse that is slowly varying with propagation, the gradient of the dipole contribution, as well as the Gouy phase, can be neglected, such that the phase mismatch is given by the wave-vector difference of the 
driving laser and HHG radiation (see SM [48] for further discussion). For reasonably high harmonic orders $q$, the XUV dispersion can even be neglected, and the HHG phase-matching only depends on the wave vector of the driving laser pulse alone. The neutral atom contribution is negative and can be balanced by the positive plasma contribution, depending on the fraction of ionized gas atoms $\eta$. At the critical ionization fraction $\eta_{\mathrm{cr}}$, both terms exactly cancel, and perfect phase-matching occurs [28]. For a given pulse shape and driving wavelength, it is reached at a specific intensity, which in turn limits the maximum HHG photon energy. This energy is the aforementioned phasematching cutoff [28]. For argon at a wavelength around $800 \mathrm{~nm}$, the critical density is $3.8 \%$, and it is reached with our pulse at $3.3 \times 10^{14} \mathrm{~W} / \mathrm{cm}^{2}$, which would give a phasematched cutoff of $70 \mathrm{eV}$. However, in our experiment, we observe an XUV cutoff of around $80 \mathrm{eV}$. In order to understand our results, we therefore have to additionally consider the phase-matching contribution of the HHG dipole.

This reasoning is further motivated by Fig. 2(d), which shows the simulated maximum intensity of the pulse on the propagation axis for HHG in argon (red) and neon (black). The pressure distribution is shown as the blue-shaded area. While in neon the intensity profile closely follows the Gaussian beam, consistent with our assumption of approximately linear propagation, the pulses are strongly modified in argon. As soon as the pressure rises, there is a strong drop in intensity from $4.7 \times 10^{14} \mathrm{~W} / \mathrm{cm}^{2}$ to $3 \times$ $10^{14} \mathrm{~W} / \mathrm{cm}^{2}$ within the first few $100 \mu \mathrm{m}$ of the target, followed by a slower decrease. This behavior can be explained by the different ionization fractions for the two gases, which is illustrated in Fig. 2(e). The fraction of ionized atoms (dashed lines) after the pulse has passed is more than a factor 100 higher at the beginning of the target for argon (up to 50\%) compared to neon (up to $0.4 \%$ ), which is due to the difference in ionization potentials $\left(I_{\mathrm{p}, \text { argon }}=15.8 \mathrm{eV}\right.$ and $\left.I_{\mathrm{p} \text {,neon }}=21.6 \mathrm{eV}\right)$ and the highly nonlinear ionization probability in the tunneling regime. At these pressures, the plasma generation in argon influences the pulse intensity through absorption and defocusing, which leads to the strong intensity decay on axis. Moreover, the pulse experiences a blueshift since longer wavelengths are affected more by plasma defocusing. We note that due to the geometry of our beamline, which blocks the driving laser on the propagation axis, this reshaping is only very weakly present in the measured streaking curve, as confirmed by a full simulation of the HHG process, beamline propagation, and refocusing in the streaking focus (see SM [48] for discussion). The high ionization probability also influences the XUV generation and leads to an increase in the HHG source term (solid line), which is proportional to the ionization probability amplitude. While the source term in the $80-\mathrm{eV}$ window in neon (black solid line) stays almost constant throughout the target, there is a strong peak at the target entrance for argon (red solid line), which decays within a few $100 \mu \mathrm{m}$ as the intensity decreases and the HHG cutoff falls below the considered energy window. Thus, for argon, in the region where the XUV generation takes place, the pulses get strongly modified in intensity and driving wavelength, affecting phase-matching, which we have to account for.

\section{Complete phase-matching description and comparison to experiment}

We introduce here a straightforward application of Eq. (1), which explicitly contains the effect of the blueshift on the dipole contribution, to describe the phase mismatch:

$$
\begin{aligned}
\Delta k(t, z)= & -q \partial_{z} \phi_{\mathrm{IR}}(t, z) \\
& +\alpha U_{p} / \hbar \omega_{0}\left(\frac{\partial_{z} I(t, z)}{I}-3 \frac{\partial_{z} \omega_{0}(t, z)}{\omega_{0}}\right),
\end{aligned}
$$

with the phase of the driving laser field $\phi_{\mathrm{IR}}$, the instantaneous intensity $I(t, z)$, and radial driving frequency $\omega_{0}(t, z)$. All values are calculated in the comoving reference frame in our simulation. Together with the assumption that the XUV propagates with the speed of light, this is the reason that the XUV wave-vector contribution does not appear in Eq. (2). Since we investigate the phase mismatch close to the cutoff at $80 \mathrm{eV}$, we set $\alpha=3.2$. In principle, for a fixed XUV energy, $\alpha$ is also dependent on the intensity and laser frequency, which we ignore for simplicity. As a consequence, the phase mismatch is split into two contributions, one from the driving laser pulse and the other from the XUV dipole. We would like to point out that, by using the numerical calculation for the driving laser-pulse phase, the first term intrinsically contains atomic and plasma dispersion as well as any geometric phase.

With this expression, we analyze the phase-matching for argon at the entrance of the HHG target $(z=-0.8 \mathrm{~mm})$, at the maximum of the XUV source term. Figure 3(a) shows the driving laser field (red solid line) and the fraction of ionized atoms (black solid line). Already at the beginning of the time window investigated here, the fraction of ionized atoms is $4.8 \%$, and therefore, it is above the critical ionization of 3.8\%. Figure 3(b) shows the phase mismatch calculated from different expressions (solid and dashed lines) and the XUV source term amplitude in the energy region around $80 \mathrm{eV}$ (blue shaded area). The XUV source term exhibits two main peaks of almost equal height from two different half-cycles at around $-0.1 \mathrm{fs}$ and $1.2 \mathrm{fs}$, similar to Fig. 2(c). All phase-matching expressions show the general trend of an increase of the phase mismatch as the plasma density increases over consecutive half-cycles, which qualitatively explains why the second peak is more strongly suppressed compared to the first one. However, the expressions neglecting the dipole contribution [solid gray line, Eq. (2) of the SM [48], and dashed black line, Eq. (2) 

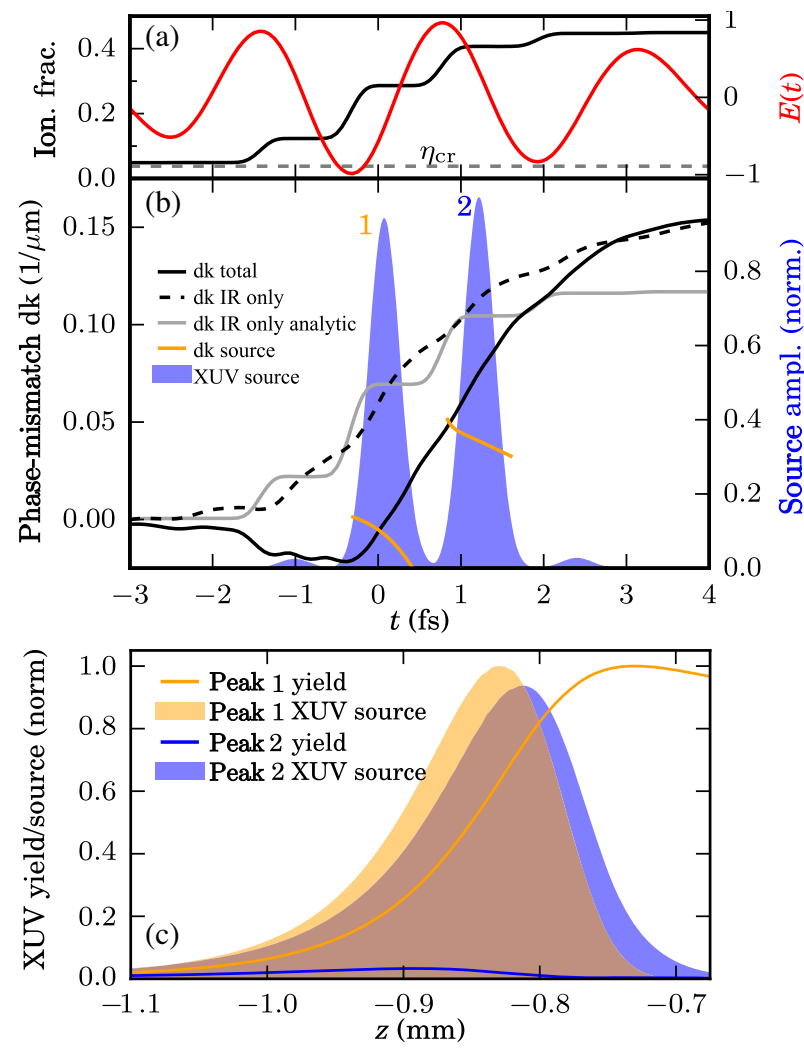

FIG. 3. On-axis transient phase-matching and XUV generation in argon. (a) Driving laser pulse (red) and the time-dependent fraction of ionized atoms (black). (b) On-axis phase mismatch in the analytic expression without a dipole (gray), the calculated phase mismatch with the dipole contribution (black) and without (black dashed), and the numerical phase mismatch calculated from the complex source term (orange) and the source-term amplitude (blue shaded area) at $z=-0.8 \mathrm{~mm}$. (c) On-axis source-term amplitude (shaded area) and evolution of the XUV amplitude from the full simulation (solid line). The color indicates the first (orange) or second (blue) half-cycle centered around $0.1 \mathrm{fs}$ and $1.2 \mathrm{fs}$, respectively, as shown in panel (b).

without a dipole term, in Fig. 3(b)] also exhibit a strong phase mismatch for the first peak and an overall small difference of the phase mismatch of both peaks. In comparison, the expression that includes the dipole term (solid black line) shows a smaller phase mismatch at the beginning of the pulse up to around $1.5 \mathrm{fs}$ and a zero crossing of the phase mismatch for the first peak. This expression can be explained by closer examination of Eq. (2). As the plasma density rises above $\eta_{\mathrm{cr}}$, the phase mismatch due to the propagation of the driving laser increases [first term in Eq. (2), see also Eq. (1)]. However, since ionization, plasma formation, and plasma defocusing generally lead, on axis, to an intensity decay $\left(\partial_{z} I<0\right)$ and a blueshift $\left(\partial_{z} \omega>0\right)$, the last term is negative and can counteract the plasma dispersion. In other words, the plasma-induced increase of the driving laserpulse phase is balanced by a decrease of the XUV dipole phase. Hence, phase-matching can be achieved even above $\eta_{\mathrm{cr}}$, and higher cutoff energies than predicted by the phasematching cutoff are possible [36]. Pulse reshaping therefore can enable phase-matching at least over small distances, as pointed out in Refs. [31-33,36,43], which is in contrast to the general notion for longer generation lengths - that the driving laser pulse should ideally remain unchanged (see, e.g., Ref. [24]).

In order to evaluate this phase-matching expression, we compare it to the phase mismatch numerically calculated from the XUV source term (solid orange line; see Sec. IV for details). Since we assume an XUV phase velocity of $c$ and are in a comoving frame of reference, the numerical phase mismatch is simply given by the derivative of the source-term phase along the propagation axis. It is apparent that the numerically calculated value is only close to the full expression [Eq. (2)] and strongly deviates from the other two. However, the numerical expression shows the opposite tilt compared to the analytical expression. The main reason is that we assume a constant $\alpha$, while in reality, it increases from long to short trajectories, i.e., from earlier to later rescattering times. This increase could be taken into account by calculating the instantaneous frequency and the corresponding instantaneous dipole phase coefficient. Better agreement could probably be obtained by expressions that contain the SFA integrals $[32,33,43]$, however, at the expense of drastically increased complexity, comparable to a full SFA HHG calculation. We conclude that Eq. (2) gives a good quantitative estimate for the value of the actual phase mismatch. The result of the phase mismatch for the two different peaks of the XUV source term in Fig. 3(b) for the XUV buildup on the propagation axis is shown in Fig. 3(c). While the strength of the source term (shaded area) of both peaks is similar, the resulting XUV yield for the first peak (orange line) is much higher than for the second (blue line). This result is an immediate consequence of the plasma-induced transient phase mismatch discussed above. After the generation of the harmonic at the entrance, reabsorption in the target sets in. For the conditions in our simulations, XUV photons generated at the entrance of the target are transmitted with a probability of roughly 0.5 through the target for argon.

\section{E. Overdriven regime for different wavelengths and gases}

Extension of the effects of pulse reshaping, intensity decay, and blueshift on HHG phase-matching becomes more important for the longer wavelength drivers, where HHG often requires tight focusing of few-cycle pulses with high gas pressure [37] to generate XUV pulses in the water window $[25,26,37,53]$. To illustrate this case, we extend our simulations to these conditions, including driving laser wavelengths up to $1.9 \mu \mathrm{m}$ and for different HHG target gases. To mimic the experimental situation in Ref. [37], 

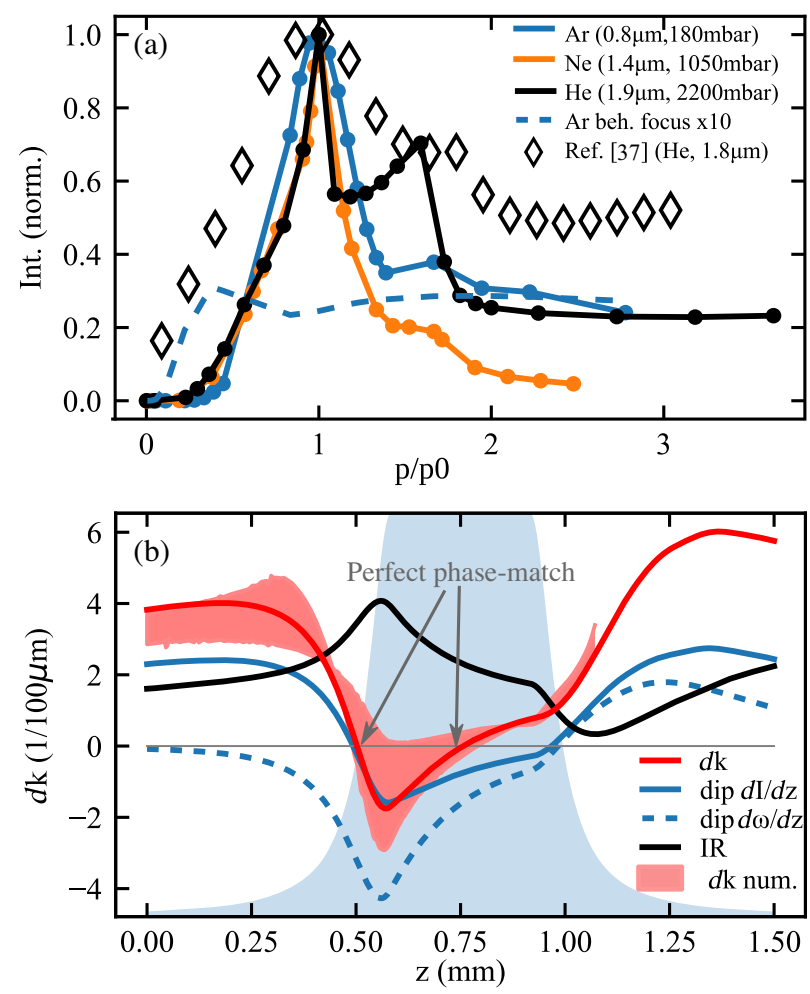

FIG. 4. Simulated high-harmonic generation dynamics in the tight focusing regime for different wavelengths and gases. (a) Normalized far-field XUV flux in the cutoff region versus normalized pressure for a 1.75-cycle pulse at $800 \mathrm{~nm}$ in argon (solid blue line, $p_{0}=180 \mathrm{mbar}, 75-95 \mathrm{eV}, w_{0}=20 \mu \mathrm{m}$ ), at $1400 \mathrm{~nm}$ in neon (solid orange line, $p_{0}=1050$ mbar, 250$320 \mathrm{eV}, w_{0}=35 \mu \mathrm{m}$ ), and $1900 \mathrm{~nm}$ in helium (solid black line, $p_{0}=2000$ mbar, 560-700 eV, $w_{0}=47.5 \mu \mathrm{m}$ ). Here, the target is positioned in front of the focus. The flux in argon for a gas target positioned after the focus is shown as a dashed blue line (upscaled by a factor 10). We compare the helium simulations to the data from Ref. [37] (open diamonds). (b) Different contributions to the total phase mismatch (thick red line) on axis for the most intense XUV burst for argon at $p=p_{0}$ calculated by Eq. (2): The phase-mismatch IR is due to the propagation of the laser driving pulse (black line), dip; $d I / d z$ and dip; and $d \omega / d z$ from the HHG dipole caused by the change of laser intensity (solid blue line) and the blueshift of the laser pulse (dashed blue line), respectively. Moreover, the numerically calculated total phase mismatch within the half-cycle of the XUV burst is shown as the red shaded area. The blue shaded area indicates the gas pressure distribution.

here, we place the target in front of the focus, and the intensity at the target entrance is chosen such that 10 times the critical ionization would be reached at the central halfcycle of the pulse in free-space propagation.

The normalized XUV flux for different HHG generation conditions is shown in Fig. 4(a). The XUV or soft-X-ray flux at different static pressures is analyzed within energy windows of 75-95 eV, 250-320 eV, and 550-650 eV for argon, neon, and helium, respectively. The driving laser wavelengths of the 1.75 -cycle pulses are $0.8 \mu \mathrm{m}, 1.4 \mu \mathrm{m}$, and $1.9 \mu \mathrm{m}$, respectively. The focusing conditions and gas target dimensions scale with wavelength (see Sec. IV for details). The flux is integrated over a divergence angle of approximately $1.5 \mathrm{mrad}$. Simulations are carried out only for a single CEP $(=0)$. The $x$ axis is normalized to the pressure $p_{0}$ of maximum yield. All three curves show a similar behavior. After an initial strong increase below the maximum, the flux rapidly drops again but then stabilizes at a level depending on the gas type. This result is consistent with the calculation and analysis in Ref. [33]. There is an oscillation observable after the first maximum, which becomes very prominent for helium, as discussed below. The approximately constant level at high pressures can be qualitatively explained by the increasing localization of the source contribution at the entrance of the target, as also observed in the simulations above [Figs. 2(e) and 3(c)], and the transmission factors through the gas target for the respective energy window at $p_{0}$, which is around 0.5 for neon, 0.8 for argon, and 0.98 for helium. For comparison, we show the flux for argon (dashed blue line upscaled by a factor of 10), when the target is placed at a position half the Rayleigh length behind the focus for an even increased intensity that corresponds to $15 \eta_{\mathrm{cr}}$. There, the maximum flux occurs at a lower pressure and is almost 2 orders of magnitude smaller. Additionally, we show the data of Ref. [37] for helium (open diamonds, extracted at $3.3 \mathrm{bar}, 410-700 \mathrm{eV}$ ), which agree qualitatively very well with our simulations.

The maximum XUV or soft-X-ray flux is reached for specific phase-matching conditions. Figure 4(b) shows the individual contributions to the on-axis phase-matching according to Eq. (2) for the peak of the main XUV pulse at $p_{0}$. The presented data are for argon, but the conditions for neon and helium are almost identical (see SM [48]). The contribution of the driving pulse (black solid line) is positive throughout the target due to the strong plasma contribution. The HHG dipole term due to the intensity change (blue solid line) reflects the derivative of the intensity. First, it is positive, followed by a change in sign slightly before the full pressure is reached; it then increases again after the target. On the contrary, the dipole term due to the blueshift (blue dashed line) is negative before and within the target and then switches to positive at the end of the target. We want to point out that the blueshift dipole term dominates here, and it is absolutely crucial for the understanding of the total phase mismatch. Since the dipole terms counteract the driving laser contribution to the phase mismatch, the overall phase mismatch (red solid line) is close to zero within the gas target. Indeed, there is a zero crossing slightly before the entrance and then close to the middle of the gas target. The zero crossing at the target entrance is located here in the rising edge of the pressure distribution and seems to occur for a wide range of pressures. This finding explains the results of Ref. [37], 
which observed an XUV buildup at the target entrance in their simulations. We compare the semianalytic to the numerical expression (red shaded area), and again, very good agreement is observed (for a further discussion, see SM [48]). At the condition of maximum flux, shown here, while starting to localize towards the target entrance, the source term still extends over the whole target length.

In order to explain the oscillation after the maximum observed in Fig. 4(a), we have to investigate what happens with rising pressure: The maximum of the XUV source term becomes increasingly localized at the entrance; therefore, the second zero crossing of the phase mismatch does not contribute to XUV buildup, and at the same time, the XUV light is absorbed by the target. We note that while the phase-mismatch curve also slightly changes, it qualitatively stays the same in this pressure range. Therefore, the flux drops rapidly after the maximum. As the pressure rises further, the source-term maximum eventually overlaps with the first zero crossing of the phase mismatch, which leads to a small secondary maximum. The pressure of maximum flux depends on the energy window, and we observe that for lower photon energies, higher pressures are required, as evident in the data of Ref. [37]. In principle, the mechanism discussed would allow us to transiently phase-match very high photon energies; however, due to the strong intensity clamping at the conditions of maximized photon flux, the HHG cutoff lies in the region of the classical phasematching cutoff.

Again, since we investigate the on-axis flux in the far field, while for certain conditions off-axis phase-matching might be favorable [35,36], we have limited ourselves to the analysis given above. We want to point out that, unlike in Ref. [35], short trajectory contributions dominate the plateau region of the HHG spectrum, even though the gas target is placed in front of the focus, consistent with Ref. [37]. This difference can be explained by the blueshift dipole term, which is stronger for few-cycle lasers. Similarly to Ref. [35], we observe the formation of a flat-top electric-field amplitude and a flat wavefront for the main cycle, due to plasma defocusing, which should also lead to improved divergence properties of the emitted harmonics. Since under the pressure conditions of maximum flux and above, ionization and plasma-induced phasematching change drastically between different half-cycles for few-cycle lasers, transient phase-matching will play an important role, as discussed for our experiments above. Moreover, even though we restrict ourselves to the analysis of the longitudinal on-axis phase-matching, Eq. (2) can easily be generalized to $3 \mathrm{D}$, if, for example, divergenceangle-resolved HHG spectra are studied.

In order to demonstrate that these conditions allow isolated attosecond pulse generation in the overdriven regime, we investigate the CEP-dependent spectra for helium in Fig. 5(a). The parameters are the same as above but scaled to $1.8 \mu \mathrm{m}$ at 2000 mbar helium. The
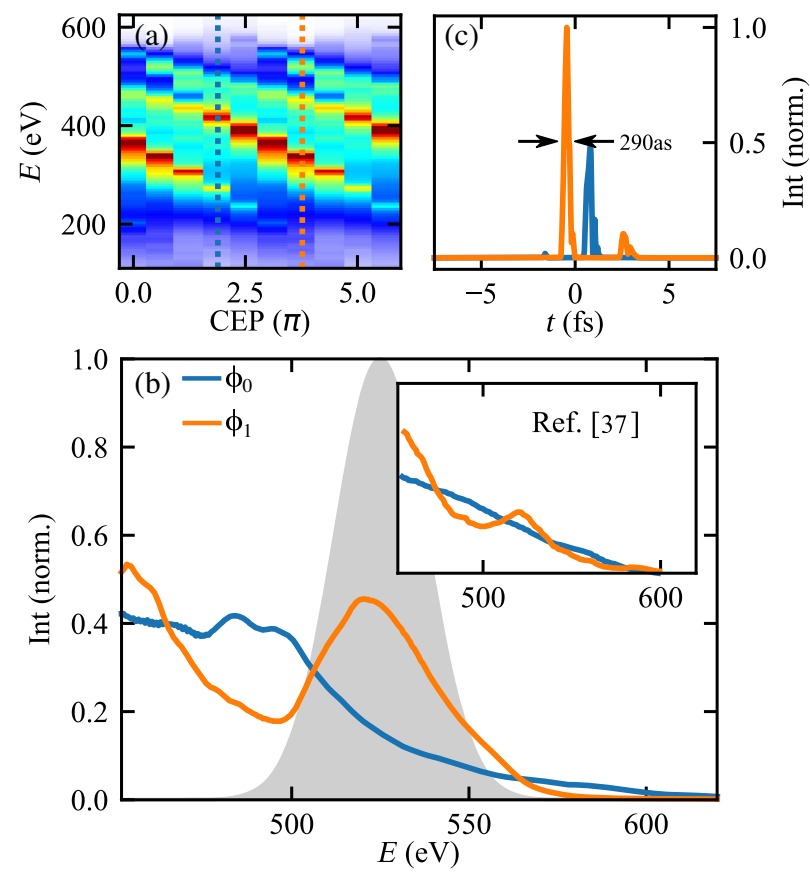

FIG. 5. Simulated CEP variation and isolated attosecond pulse generation for helium for the same conditions as in Fig. 4 but scaled to a driving wavelength of $1800 \mathrm{~nm}$ and at $p=2000$ mbar. (a) CEP-resolved spectra. Transmission through a $0.1-\mu \mathrm{m}$ aluminum foil has been included to filter out the low-energy contribution. The color bar is the same as in Fig. 1. (b) XUV spectra near the cutoff for two different CEP values $\phi_{0}$ and $\phi_{1}$ as indicated in panel (a). The inset shows the data from Ref. [37]. The isolated attosecond pulses, generated in the energy window illustrated by the gray area, are shown in panel (c).

transmission of a $0.1-\mu \mathrm{m}$ aluminum filter has been added. Overall, a strong CEP dependence is observed. The spectra in the cutoff region for two specific CEP values are depicted in Fig. 5(b). They have a remarkable resemblance to the experimental data of Ref. [37], shown in the inset. The gray shaded area indicates the bandwidth for which the resulting soft-x-ray pulse, shown in Fig. 5(c), is calculated. Indeed, a very strong isolated pulse is observed. Even in the complementary phase, an almost isolated pulse is generated. The pulse is slightly positively chirped and therefore longer than the Fourier limit. If energy windows that cover almost the whole spectrum are used in experiments, the suitability of the generated pulses depends on which contrast between the main soft-x-ray burst and secondary bursts is acceptable. As can be seen in Fig. 5(a) from the half-cycle cutoffs - manifested as diagonal stripes - several half-cycles will contribute to the resulting pulses if, e.g., all energies above $280 \mathrm{eV}$ are included. Nevertheless, for most CEP values, there is always one half-cycle that clearly dominates the spectrum and therefore allows isolated attosecond pulse generation with a moderate contribution of secondary pulses. 


\section{CONCLUSION}

We have presented an experimental and numerical study of the generation of isolated attosecond pulses in argon and neon through high-harmonic generation at $80 \mathrm{eV}$. To our knowledge, this value is the highest photon energy for which isolated attosecond pulses in argon driven by laser pulses in the $800-\mathrm{nm}$ wavelength region have been reported, and it is clearly above the classical phasematching cutoff. Both attosecond XUV and driving laser pulses were characterized through attosecond streaking. While the generation of IAPs in neon was consistent with amplitude gating, we find that ionization-induced transient phase-matching in the overdriven regime is the underlying gating mechanism in argon. In numerical simulations, we observe that the high ionization of the medium leads to a rapid decrease of the peak intensity, thereby limiting the HHG to the first few $100 \mu \mathrm{m}$ in argon. Analyzing the XUV source term, we find that, due to the high ionization rate, the single-atom HHG process is significantly enhanced compared to neon. We compare different analytic phasematching expressions, and through comparison with the numerical calculation, we find that only the extended expression introduced here, which contains the effect of the blueshift on the HHG dipole, provides a quantitative description. We show that the rapid decrease of the intensity, as well as the blueshift, significantly contributes to the phase-matching close to the XUV cutoff, allowing phase-matched XUV generation for a single half-cycle at considerably higher ionization fractions compared to slowly varying intensity conditions.

We also perform simulations for other gas types and wavelengths for conditions that are relevant to other recent experiments, and we analyze the pressure-dependent XUV flux close to the cutoff. At the maximum flux, phasematching is strongly influenced through blueshift and intensity decay due to plasma generation. We find that placing the target in front of the focus allows significantly higher flux under these conditions, as the beam convergence and plasma defocusing balance each other. Moreover, our results show how pulse reshaping influences the phase-matching under high-pressure and strong focusing conditions. These findings will play a vital role for the analysis of current and future experiments that aim at XUV generation in the water window and beyond, which in turn promises many interesting applications.

\section{METHODS}

\section{A. Experimental optimization of the HHG process}

Experimentally, we start with HHG in neon by first optimizing the gas pressure for maximum XUV flux. Note that due to the feedback loop of the automated gas valve from the background pressure in the HHG chamber, only a rough control is possible; however, our results are not dependent on the exact value of the pressure (see SM [48] for further discussion). We then continued by finely adjusting the fused silica wedge insertion for maximum CEP dependence in the cutoff region of the XUV spectrum. Subsequently, a streaking spectrogram in neon is recorded by inserting the double mirror. We then switch to argon after evacuating the gas supply line. Besides gas pressure and dispersion, we also change the position of the focusing mirror as indicated in Sec. II A and optimize the flux, stability, and CEP dependence in the XUV spectrum. Originally, as inferred from plasma generation in lowpressure air, the HHG gas target had been placed several $\mathrm{mm}$ behind the focus. With this procedure, we end up with a shift of the focusing mirror by about $5.5 \mathrm{~mm}$ towards the target, roughly an additional $260 \mu \mathrm{m}$ of fused silica and similar flow rates. Afterwards, again the streaking spectrograms are recorded.

\section{B. Simulation model}

The simulation code is based on a model used in previous publications [51]. The spot size on the mirror focusing into the HHG target is $7 \mathrm{~mm}$. The HHG target is assumed to have a thickness of $1.5 \mathrm{~mm}$ filled with $150 \mathrm{mbar}$ of either argon or neon. The pressure gradient outside the target is taken from Ref. [37]. From the focal length, pulse energy, and waveform, as well as the generated XUV spectra, we estimate a peak intensity of $5.35 \times 10^{14} \mathrm{~W} / \mathrm{cm}^{2}$ for the neon input pulse. This estimation results in $4.8 \times 10^{14} \mathrm{~W} / \mathrm{cm}^{2}$ for argon. The shift of the focusing mirror $(f=75 \mathrm{~cm})$ is taken care of by placing the entrance of the HHG gas target for neon $5.5 \mathrm{~mm}$ after the target and at the focus for argon. The gas targets are modeled with a Lorentzian pressure decay along the propagation axis from the entrance and exit, as in previous publications [31,37].

The pulse obtained at the end of the HHG target is propagated through the beamline by using the propagator of the Helmholtz equation in the paraxial approximation. The beam parameters are calculated at the positions of the iris (distance between HHG target to iris: $d_{\mathrm{HHG}-\mathrm{iris}}=1.3 \mathrm{~m}$ ), the $\mathrm{Zr}$ filter $\left(d_{\text {iris-filter }}=0.3 \mathrm{~m}\right)$ that spatially separates XUV and the driving laser, the focusing double mirror $\left(d_{\mathrm{HHG}-\text { double mirror }}=2.0 \mathrm{~m}\right.$; radius: $r_{\text {inner XUV mirror }}=3 \mathrm{~mm}$, $r_{\text {outer IR mirror }}=12.5 \mathrm{~mm} ;$ focal length: $f_{\text {double mirror }}=$ $125 \mathrm{~mm})$, and finally in the focus $\left(d_{\text {double mirror-focus }}=\right.$ $133 \mathrm{~mm}$ ) [see Fig. 1(a)]. The system is assumed to be optically centered in order to employ cylindrical symmetry, which neglects the fact that the focusing double mirror is slightly tilted (see SM [48] for more details).

\section{Calculation of the time-dependent XUV source term}

In the paraxial approximation, the propagation of a pulse is given by

$$
\frac{\partial E}{\partial z}-\mathrm{i}\left[k(\omega)-\omega / v_{\mathrm{g}}\right] E-\frac{\mathrm{i}}{2 k(\omega)} \Delta_{\perp} E=\frac{\mathrm{i} \omega}{2 n(\omega) \mathrm{c}} \frac{P}{\epsilon_{0}},
$$


where $k(\omega)$ is the wave vector, $v_{\mathrm{g}}$ is the group velocity, $\Delta_{\perp}$ is the Laplacian of the transverse coordinates, $n(\omega)$ is the refractive index, $\epsilon_{0}$ is the permittivity of free space, and $P$ is the nonlinear polarization. Note that we perform the calculation in the comoving frame of reference. The term on the right-hand side is the source term. In the absence of dispersion (second term $=0$ ) and neglecting diffraction (third term $=0$ ), it directly describes the change of the electric field. For the time-dependent XUV-source term, we use the right-hand side of the above equation, multiply it by $\exp \left(-2 \ln (2)[(\hbar \omega-80(\mathrm{eV})) / 6.4(\mathrm{eV})]^{2}\right)$ and perform an inverse Fourier transform. With the source-term intensity, the squared source term is denoted by source-term intensity. When referring to the integrated source term, we mean the integration of the absolute value of the source term.

\section{ACKNOWLEDGMENTS}

We are grateful for support from the King-Saud University in the framework of the MPQ-KSU-LMU Collaboration. We thank Ferenc Krausz for his support and fruitful discussions. The MPQ/LMU group acknowledges support from the DFG via the Munich Centre for Advanced Photonics. J. S. acknowledges support from the Max Planck Society via the IMPRS-APS. The KSU group acknowledges support from the King Saud University, Riyadh, Saudi Arabia via the Researchers Supporting Project RSP-2019/152. M.F. K. is grateful for support from the Max Planck Society and from the DFG via Grant No. KL1439/10-1. S. B. is grateful for support by the Alexander von Humboldt Foundation.

The authors declare no competing financial interest.

M. F. K. and A. M. A. conceived the experiment. J. S., B. F., W. S., I. L., H. A. M., A. M. K., and M. A. conducted the experiments. J. S. analyzed the data and developed the theoretical simulations based on code by M. H. and I. P.; C. J., N. G. K., and T. P-C. helped with the installation of the attosecond beamline and supported initial experiments. J.S. wrote the manuscript with support from M. F. K. and S. B., and it was reviewed by all authors.

[1] A. McPherson, G. Gibson, H. Jara, U. Johann, T. S. Luk, I. A. McIntyre, K. Boyer, and C. K. Rhodes, Studies of Multiphoton Production of Vacuum-Ultraviolet Radiation in the Rare Gases, J. Opt. Soc. Am. B 4, 595 (1987).

[2] M. Ferray, A. L'Huillier, X. F. Li, L. A. Lompré, G. Mainfray, and C. Manus, Multiple-Harmonic Conversion of 1064 nm Radiation in Rare Gases, J. Phys. B 21, L31 (1988).

[3] X. F. Li, A. L'Huillier, M. Ferray, L. A. Lompré, and G. Mainfray, Multiple-Harmonic Generation in Rare Gases at High Laser Intensity, Phys. Rev. A 39, 5751 (1989).

[4] Y. Liang, S. Augst, S. L. Chin, Y. Beaudoin, and M. Chaker, High Harmonic Generation in Atomic and Diatomic
Molecular Gases Using Intense Picosecond Laser PulsesA Comparison, J. Phys. B 27, 5119 (1994).

[5] P. M. Paul, E. S. Toma, P. Breger, G. Mullot, F. Augé, P. Balcou, H. G. Muller, and P. Agostini, Observation of a Train of Attosecond Pulses from High Harmonic Generation, Science 292, 1689 (2001).

[6] M. Hentschel, R. Kienberger, C. Spielmann, G. A. Reider, N. Milosevic, T. Brabec, P. Corkum, U. Heinzmann, M. Drescher, and F. Krausz, Attosecond Metrology, Nature (London) 414, 509 (2001).

[7] F. Krausz and M. Ivanov, Attosecond Physics, Rev. Mod. Phys. 81, 163 (2009).

[8] L. Gallmann, C. Cirelli, and U. Keller, Attosecond Science: Recent Highlights and Future Trends, Annu. Rev. Phys. Chem. 63, 447 (2012).

[9] F. Calegari, G. Sansone, S. Stagira, C. Vozzi, and M. Nisoli, Advances in Attosecond Science, J. Phys. B 49, 062001 (2016).

[10] I. Orfanos, I. Makos, I. Liontos, E. Skantzakis, B. Förg, D. Charalambidis, and P. Tzallas, Attosecond Pulse Metrology, APL Photonics 4, 080901 (2019).

[11] P. B. Corkum, Plasma Perspective on Strong Field Multiphoton Ionization, Phys. Rev. Lett. 71, 1994 (1993).

[12] G. Steinmeyer, D. Sutter, L. Gallmann, N. Matuschek, and U. Keller, Frontiers in Ultrashort Pulse Generation: Pushing the Limits in Linear and Nonlinear Optics, Science 286, 1507 (1999).

[13] T. Brabec and F. Krausz, Intense Few-Cycle Laser Fields: Frontiers of Nonlinear Optics, Rev. Mod. Phys. 72, 545 (2000).

[14] G. Sansone, E. Benedetti, F. Calegari, C. Vozzi, L. Avaldi, R. Flammini, L. Poletto, P. Villoresi, C. Altucci, R. Velotta, S. Stagira, S. De Silvestri, and M. Nisoli, Isolated SingleCycle Attosecond Pulses, Science 314, 443 (2006).

[15] H. Mashiko, S. Gilbertson, M. Chini, X. Feng, C. Yun, H. Wang, S. D. Khan, S. Chen, and Z. Chang, Extreme Ultraviolet Supercontinua Supporting Pulse Durations of Less than One Atomic Unit of Time, Opt. Lett. 34, 3337 (2009).

[16] P. Tzallas, E. Skantzakis, C. Kalpouzos, E. Benis, G. D. Tsakiris, and D. Charalambidis, Generation of Intense Continuum Extreme-Ultraviolet Radiation by Many-Cycle Laser Fields, Nat. Phys. 3, 846 (2007).

[17] T. Pfeifer, A. Jullien, M. J. Abel, P. M. Nagel, L. Gallmann, D. M. Neumark, and S. R. Leone, Generating Coherent Broadband Continuum Soft-X-Ray Radiation by Attosecond Ionization Gating, Opt. Express 15, 17120 (2007).

[18] F. Ferrari, F. Calegari, M. Lucchini, C. Vozzi, S. Stagira, G. Sansone, and M. Nisoli, High-Energy Isolated Attosecond Pulses Generated by Above-Saturation Few-Cycle Fields, Nat. Photonics 4, 875 (2010).

[19] E. Constant, D. Garzella, P. Breger, E. Mével, C. Dorrer, C. Le Blanc, F. Salin, and P. Agostini, Optimizing High Harmonic Generation in Absorbing Gases: Model and Experiment, Phys. Rev. Lett. 82, 1668 (1999).

[20] S. Kazamias, D. Douillet, F. Weihe, C. Valentin, A. Rousse, S. Sebban, G. Grillon, F. Augé, D. Hulin, and P. Balcou, Global Optimization of High Harmonic Generation, Phys. Rev. Lett. 90, 193901 (2003). 
[21] A. S. Sandhu, E. Gagnon, A. Paul, I. Thomann, A. Lytle, T. Keep, M. M. Murnane, H. C. Kapteyn, and I. P. Christov, Generation of Sub-Optical-Cycle, Carrier-Envelope-Phase Insensitive, Extreme-UV Pulses via Nonlinear Stabilization in a Waveguide, Phys. Rev. A 74, 061803(R) (2006).

[22] M. J. Abel, T. Pfeifer, P. M. Nagel, W. Boutu, M. J. Bell, C. P. Steiner, D. M. Neumark, and S. R. Leone, Isolated Attosecond Pulses from Ionization Gating of HighHarmonic Emission, Chem. Phys. 366, 9 (2009).

[23] I. Thomann, A. Bahabad, X. Liu, R. Trebino, M. Murnane, and H. Kapteyn, Characterizing Isolated Attosecond Pulses from Hollow-Core Waveguides Using Multi-cycle Driving Pulses, Opt. Express 17, 4611 (2009).

[24] M.-C. Chen, C. Mancuso, C. Hernández-García, F. Dollar, B. Galloway, D. Popmintchev, P.-C. Huang, B. Walker, L. Plaja, A. A. Jaron-Becker et al., Generation of Bright Isolated Attosecond Soft X-Ray Pulses Driven by Multicycle Midinfrared Lasers, Proc. Natl. Acad. Sci. U.S.A. 111, E2361 (2014).

[25] T. Popmintchev, M.-C. Chen, D. Popmintchev, P. Arpin, S. Brown, S. Ališauskas, G. Andriukaitis, T. Balčiunas, O. D. Mücke, A. Pugzlys, A. Baltuška, B. Shim, S. E. Schrauth, A. Gaeta, C. Hernández-García, L. Plaja, A. Becker, A. Jaron-Becker, M. M. Murnane, and H. C. Kapteyn, Bright Coherent Ultrahigh Harmonics in the keV X-Ray Regime from Mid-Infrared Femtosecond Lasers, Science 336, 1287 (2012).

[26] S. M. Teichmann, F. Silva, S. L. Cousin, M. Hemmer, and J. Biegert, 0.5-keV Soft X-Ray Attosecond Continua, Nat. Commun. 7, 11493 (2016).

[27] M. B. Gaarde, J. L. Tate, and K. J. Schafer, Macroscopic Aspects of Attosecond Pulse Generation, J. Phys. B 41, 132001 (2008).

[28] T. Popmintchev, M.-C. Chen, O. Cohen, M. E. Grisham, J. J. Rocca, M. M. Murnane, and H. C. Kapteyn, Extended Phase Matching of High Harmonics Driven by Mid-Infrared Light, Opt. Lett. 33, 2128 (2008).

[29] T. Popmintchev, M.-C. Chen, A. Bahabad, M. Gerrity, P. Sidorenko, O. Cohen, I. P. Christov, M. M. Murnane, and H. C. Kapteyn, Phase Matching of High Harmonic Generation in the Soft and HardX-Ray Regions of the Spectrum, Proc. Natl. Acad. Sci. U.S.A. 106, 10516 (2009).

[30] C. Hernández-García, T. Popmintchev, M. Murnane, H. Kapteyn, L. Plaja, A. Becker, and A. Jaron-Becker, Isolated Broadband Attosecond Pulse Generation with Near-and Mid-Infrared Driver Pulses via Time-Gated Phase Matching, Opt. Express 25, 11855 (2017).

[31] C.-J. Lai and F.X. Kärtner, The Influence of Plasma Defocusing in High Harmonic Generation, Opt. Express 19, 22377 (2011).

[32] G. Tempea, M. Geissler, M. Schnürer, and T. Brabec, SelfPhase-Matched High Harmonic Generation, Phys. Rev. Lett. 84, 4329 (2000).

[33] V. S. Yakovlev, M. Ivanov, and F. Krausz, Enhanced PhaseMatching for Generation of Soft X-Ray Harmonics and Attosecond Pulses in Atomic Gases, Opt. Express 15, 15351 (2007).

[34] C. Liu, R. Li, Z. Zeng, Y. Zheng, P. Liu, and Z. Xu, Nonadiabatic Propagation Effect for Generating Isolated
Sub-100 as Pulses in the High-Order Harmonic Plateau, Opt. Lett. 35, 2618 (2010).

[35] C. Vozzi, M. Negro, F. Calegari, S. Stagira, K. Kovács, and V. Tosa, Phase-Matching Effects in the Generation of HighEnergy Photons by Mid-Infrared Few-Cycle Laser Pulses, New J. Phys. 13, 073003 (2011).

[36] H.-W. Sun, P.-C. Huang, Y.-H. Tzeng, J.-T. Huang, C. D. Lin, C. Jin, and M.-C. Chen, Extended Phase Matching of High Harmonic Generation by Plasma-Induced Defocusing, Optica 4, 976 (2017).

[37] A. S. Johnson, D. R. Austin, D. A. Wood, C. Brahms, A. Gregory, K. B. Holzner, S. Jarosch, E. W. Larsen, S. Parker, C. S. Strüber et al., High-Flux Soft X-Ray Harmonic Generation from Ionization-Shaped Few-Cycle Laser Pulses, Sci. Adv. 4, eaar3761 (2018).

[38] N. Ishii, K. Kaneshima, K. Kitano, T. Kanai, S. Watanabe, and J. Itatani, Carrier-Envelope Phase-Dependent High Harmonic Generation in the Water Window Using FewCycle Infrared Pulses, Nat. Commun. 5, 3331 (2014).

[39] M. Schnürer, C. Spielmann, P. Wobrauschek, C. Streli, N. H. Burnett, C. Kan, K. Ferencz, R. Koppitsch, Z. Cheng, T. Brabec, and F. Krausz, Coherent 0.5-keV X-Ray Emission from Helium Driven by a Sub-10-fs Laser, Phys. Rev. Lett. 80, 3236 (1998).

[40] E. Seres, J. Seres, F. Krausz, and C. Spielmann, Generation of Coherent Soft-X-Ray Radiation Extending Far Beyond the Titanium L Edge, Phys. Rev. Lett. 92, 163002 (2004).

[41] J. Seres, E. Seres, A. J. Verhoef, G. Tempea, C. Streli, P. Wobrauschek, V. Yakovlev, A. Scrinzi, C. Spielmann, and F. Krausz, Laser Technology: Source of Coherent Kiloelectronvolt X-Rays, Nature (London) 433, 596 (2005).

[42] E. Seres, J. Seres, and C. Spielmann, X-Ray Absorption Spectroscopy in the keV Range with Laser Generated High Harmonic Radiation, Appl. Phys. Lett. 89, 181919 (2006).

[43] M. Geissler, G. Tempea, and T. Brabec, Phase-Matched High-Order Harmonic Generation in the Nonadiabatic Limit, Phys. Rev. A 62, 033817 (2000).

[44] J. Itatani, F. Quéré, G. L. Yudin, M. Y. Ivanov, F. Krausz, and P. B. Corkum, Attosecond Streak Camera, Phys. Rev. Lett. 88, 173903 (2002).

[45] M. Kitzler, N. Milosevic, A. Scrinzi, F. Krausz, and T. Brabec, Quantum Theory of Attosecond XUV Pulse Measurement by Laser Dressed Photoionization, Phys. Rev. Lett. 88, 173904 (2002).

[46] E. Goulielmakis, M. Uiberacker, R. Kienberger, A. Baltuska, V. Yakovlev, A. Scrinzi, T. Westerwalbesloh, U. Kleineberg, U. Heinzmann, M. Drescher, and F. Krausz, Direct Measurement of Light Waves, Science 305, 1267 (2004).

[47] R. Kienberger, E. Goulielmakis, M. Uiberacker, A. Baltuska et al., Atomic Transient Recorder, Nature (London) 427, 817 (2004).

[48] See Supplemental Material at http://link.aps.org/ supplemental/10.1103/PhysRevX.10.041011 for further information on the experimental setup and results, as well as further details of the simulations and of the phase-matching analysis. 
[49] J. Schötz, B. Förg, M. Förster, W. A. Okell, M. I. Stockman, F. Krausz, P. Hommelhoff, and M. F. Kling, Reconstruction of Nanoscale Near Fields by Attosecond Streaking, IEEE J. Sel. Top. Quantum Electron. 23, 77 (2017).

[50] M. Lucchini, M. Brügmann, A. Ludwig, L. Gallmann, U. Keller, and T. Feurer, Ptychographic Reconstruction of Attosecond Pulses, Opt. Express 23, 29502 (2015).

[51] M. Högner, V. Tosa, and I. Pupeza, Generation of Isolated Attosecond Pulses with Enhancement Cavities-A Theoretical Study, New J. Phys. 19, 033040 (2017).
[52] X. M. Tong and C. D. Lin, Empirical Formula for Static Field Ionization Rates of Atoms and Molecules by Lasers in the Barrier-Suppression Regime, J. Phys. B 38, 2593 (2005).

[53] G. J. Stein, P. D. Keathley, P. Krogen, H. Liang, J. P. Siqueira, C.-L. Chang, C.-J. Lai, K.-H. Hong, G. M. Laurent, and F.X. Kärtner, Water-Window Soft $X$-Ray High-Harmonic Generation up to the Nitrogen $K$-Edge Driven by a kHz, $2.1 \mu \mathrm{m}$ OPCPA Source, J. Phys. B 49, 155601 (2016). 